\title{
Robust Aeroelastic Control of Very Flexible Wings using Intrinsic Models
}

\author{
Yinan Wang, Andrew Wynn ${ }^{\dagger}$ and Rafael Palacios ${ }^{\ddagger}$ \\ Imperial College, London SW7 2AZ, United Kingdom
}

\begin{abstract}
This paper explores the robust control of large flexible wings when their dynamics are written in terms of intrinsic variables, that is, velocities and stress resultants. Assuming 2-D strip theory for the aerodynamics, the resulting nonlinear aeroelastic equations of motion are written in modal coordinates. It is seen that a system which experiences large displacements can nonetheless be accurately described by a system with only weak nonlinear couplings in this description of the wing dynamics. As result, a linear robust controller acting on a control surface is able to effectively provide gust load alleviation and flutter suppression even when the wing structure undergoes large deformations. This is numerically demonstrated on various representative test cases.
\end{abstract}

\section{Introduction}

This paper investigates strategies for load alleviation and stabilisation of flexible wings with geometricallynonlinear deformations using conventional control surfaces. This is relevant in the development of HighAltitude Long-Endurance aircraft Unmanned Aerial Vehicles (HALE UAVs), which have seen unprecedented interest in recent years, their uses ranging from front-line military action to surveillance and observation, for example for use in the development as a low-cost alternative to communication satellites. This unique mission profile often brings design challenges unseen in other aerial vehicle designs. ${ }^{1,2}$ For example, a subclass of HALE aircraft operates on electricity provided by onboard solar panels. Sustained operation on the minimal amount of power available requires designs that are extremely light and efficient. Such designs require wings of very low compliance that are susceptible to undergoing large deformations under aerodynamic loads, particularly in the case of atmospheric turbulence and gusts. Thus, in order to realise the potential of such lightweight, flexible designs one requires the use of an advanced control system that ensure load alleviation and/or stabilisation. ${ }^{3}$

A number of integrated simulation frameworks have been constructed to model the nonlinear response to gust loads of very flexible aircraft. ${ }^{4-9}$ They have provided substantial evidence of the importance of modelling large, geometrically-nonlinear deflections, and the challenges associated to the development of control systems for gust alleviation and trajectory tracking in such systems. Cook et al. ${ }^{10}$ and Dillsaver et $a l^{11}$ applied $\mathcal{H}_{\infty}$ and LQG controllers respectively to full aircraft models and investigated the response to gust and continuous disturbances. They have highlighted the difficulty of achieving robust control on very flexible aircraft with linear control schemes when it was subject to large disturbances. The control of nonlinear structures in general has been partially reviewed by Wagg and Neild, ${ }^{12}$ with methods including adaptive control, feedback linearisation of nonlinear systems and the use of Lyapunov-based methods. In particular, Strganac ${ }^{13}$ has tackled nonlinear control by using feedback linearisation to transform a relatively small nonlinear aeroelastic system into a linear one. For larger systems, energy methods ${ }^{14}$ provide a powerful framework for nonlinear control development, however they rely on the identification on suitable Lyapunov functions of the nonlinear system. While this has been achieved for the (conservative) structural dynamics problem, ${ }^{15}$ it is still an open question how to expand this to aeroelastic systems.

Within this context, the current research will revisit the use of linear control methods on large flexible wings subject to relatively large disturbance. The framework will be the intrinsic formulation of the problem, introduced for geometriccally-exact beam dynamics by Hodges ${ }^{16}$ and expanded to nonlinear aeroelastic

*Graduate Student, Department of Aeronautics, AIAA Student Member.

$\dagger$ Lecturer, Department of Aeronautics

${ }^{\ddagger}$ Senior Lecturer, Department of Aeronautics (contact author: rpalacio@imperial.ac.uk), AIAA Member 
problems by Sotoudeh et al. ${ }^{7}$ Our description will use the modal projection of the intrinsic beam equations of Palacios, ${ }^{17}$ with aerodynamics given by the strip theory, as in Palacios and Epureanu. ${ }^{18}$ This provides a compact description of the problem suitable for control design. The paper thus describes the development of a $\mathcal{H}_{\infty}$ control system on wings described by a intrinsic modal formulation, with the aim of providing gust alleviation and disturbance rejection using conventional flap actuation. The paper will first review the geometrically exact, intrinsic formulation of beam structures, expanding the description of Palacios ${ }^{17}$ to a nonlinear cantilever beam on a moving base. Then, the 2-D aerodynamic model and the projection of the resulting nonlinear aeroelastic system on the modal basis will be discussed. Finally, the synthesis of a controller on the linearized system is outlined, followed by a presentation of a set of numerical studies of increasing complexity to illustrate the use of robust control tools in the present nonlinear aeroelastic analysis framework.

\section{Intrinsic Beam Formulation}

An intrinsic beam formulation ${ }^{16}$ uses sectional stress and velocities along to describe the dynamics of a deformable reference line with rigid cross-sections attached to each location $s$ along the line. The initial shape of this reference line is described by components of the local initial strain and curvature vector, $\gamma_{0}(s)$ and $k_{0}(s)$ respectively, in a local reference frame. The intrinsic model does not place constraints on the material properties of the beam except it must have a high aspect ratio, i.e. its sectional area being small compared to the square of the typical scale of the beam deformations. The equations of the intrinsic formulation, developed by Hodges ${ }^{16}$ will be written here as ${ }^{17,19}$

$$
\begin{aligned}
M \dot{x}_{1}-x_{2}^{\prime}-E x_{2}+\mathcal{L}_{1}\left(x_{1}\right) M x_{1}+\mathcal{L}_{2}\left(x_{2}\right) C x_{2} & =f_{1}, \\
C \dot{x}_{2}-x_{1}^{\prime}+E^{T} x_{1}-\mathcal{L}_{1}^{T}\left(x_{1}\right) C x_{2} & =0 .
\end{aligned}
$$

Dots $(\bullet)$ denote derivatives with time, $t$, while primes $\left(\bullet^{\prime}\right)$ are used for derivatives with the arc length, $s$. The first equation is the actual equation of motion, while the second is a compatibility condition between strains and velocities that ensures uniqueness of the solution. The state vector components $x_{1}(s, t) \in \mathbb{R}^{6}$ and $x_{2}(s, t) \in \mathbb{R}^{6}$ and the force vector $f_{1}(s, t) \in \mathbb{R}^{6}$ are given by

$$
x_{1}=\left(\begin{array}{c}
v \\
\omega
\end{array}\right), x_{2}=\left(\begin{array}{c}
f \\
m
\end{array}\right), f_{1}=\left(\begin{array}{c}
f_{a} \\
m_{a}
\end{array}\right),
$$

where $v(s, t)$ and $\omega(s, t)$ are the translational and angular inertial velocities; $f(s, t)$ and $m(s, t)$ are the sectional internal forces and moments; and $f_{a}(s, t)$ and $m_{a}(s, t)$ are the applied forces and moments per unit length. All vectors are expressed in their components in the local (deformed) material frame. Their definition in terms of beam displacements and rotations can be found, for example, in the work by Palacios. ${ }^{20}$ The material constants in the equation are the cross-sectional mass, $M$, and flexibility (or compliance), $C$, matrices, which can be obtained from a structural homogenisation process. ${ }^{21}$ They are full symmetric matrices that may vary with the arc length, $s$. Finally, the matrix $E$ includes the effect of the initial strain $\gamma_{0}$ and curvature $k_{0}$ by

$$
E:=\left(\begin{array}{cc}
\tilde{k}_{0} & 0 \\
\tilde{e}_{1}+\tilde{\gamma}_{0} & \tilde{k}_{0}
\end{array}\right),
$$

where $e_{1}:=(1,0,0)$ and $\tilde{\boldsymbol{n}}$ is the skew-symmetric (or cross-product) operator. The linear operators $\mathcal{L}_{1}$ and $\mathcal{L}_{2}$ are finally defined as

$$
\mathcal{L}_{1}\left(x_{1}\right):=\left(\begin{array}{cc}
\tilde{\omega} & 0 \\
\tilde{v} & \tilde{\omega}
\end{array}\right), \text { and } \mathcal{L}_{2}\left(x_{2}\right):=\left(\begin{array}{cc}
0 & \tilde{f} \\
\tilde{f} & \tilde{m}
\end{array}\right) .
$$

The intrinsic formulation is therefore defined in terms of the spatial (force) and time (velocity) derivatives of the displacements and rotations along the beam. The problem of numerically integrating (1), or otherwise characterising its solutions, needs to be solved with end conditions at $s=0$ and $s=L$, for all $t$, as well as with initial conditions for all $s$. In general, natural boundary conditions to the intrinsic formulation are posed as:

$$
\begin{aligned}
x_{1 i}(0, t) \cdot x_{2 i}(0, t) & =0 \\
x_{1 j}(L, t) \cdot x_{2 j}(L, t) & =0 .
\end{aligned}
$$


In a multi-beam configuration found on most aircraft geometries, the joints between beams can be modelled as additional boundary conditions of the type:

$$
\begin{aligned}
x_{1,1}\left(s_{1}, t\right) & =C^{1 j} x_{1, j}\left(s_{j}, t\right), \text { for } 2 \leq j \leq J \\
\sum_{j}^{J} C^{1 j} x_{2, j} & =0 .
\end{aligned}
$$

where $J$ is the number of beams in a joint and $s_{j}$ refers to the coordinate of the end of the $j$-th beam at the joint. One of the beam is used as reference, and $C^{1 j}$ is the rotation matrix from the beam $j$ in the joint to the reference one.

For the particular problem of a translating base, with $u(t) \in \mathbb{R}^{6}$ the prescribed base velocities (and angular velocities), the boundary condition becomes

$$
\begin{aligned}
& x_{1}(0, t)=u(t), \\
& x_{2}(L, t)=0 .
\end{aligned}
$$

Next, we project the system degrees of freedom into a set of mass-normalised linear normal modes (LNMs) about the undeformed beam, ${ }^{17}$

$$
\begin{aligned}
& x_{1}(s, t)=q_{1 j}(t) \phi_{1 j}(s), \\
& x_{2}(s, t)=q_{2 j}(t) \phi_{2 j}(s) .
\end{aligned}
$$

In this work, $x_{1}$ is expanded in terms of cantilever modes, according to Ref. [18, equation (19)], which are augmented by another set of 6 rigid-body modes computed from a free-free configuration which serves to contribute to $u(t)$ at $s=0$. While the resulting set is not orthogonal, it was found to simplify the numerical solution of the nonlinear system. Upon projecting onto modal coordinates using (8), (1) becomes

$$
\begin{aligned}
& A_{1} \dot{q}_{1}=B_{1} q_{2}-\left(q_{1 \ell} \Gamma_{1}^{\ell} q_{1}+q_{2 \ell} \Gamma_{2}^{\ell} q_{2}\right)+\eta_{1}, \\
& A_{2} \dot{q}_{2}=B_{2} q_{1}+q_{2 \ell}\left(\Gamma_{2}^{\ell}\right)^{\top} q_{1} .
\end{aligned}
$$

with the real coefficients

$$
\begin{aligned}
& \left(A_{1}\right)_{j k}:=\left\langle\phi_{1 j}, M \phi_{1 k}\right\rangle, \\
& \left(A_{2}\right)_{j k}:=\left\langle\phi_{2 j}, C \phi_{2 k}\right\rangle, \\
& \left(B_{1}\right)_{j k}:=\left\langle\phi_{1 j}, \phi_{2 k}^{\prime}+E \phi_{2 k}\right\rangle, \\
& \left(B_{2}\right)_{j k}:=\left\langle\phi_{2 j}, \phi_{1 k}^{\prime}-E^{T} \phi_{1 k}\right\rangle, \\
& \left(\Gamma_{1}^{\ell}\right)_{j k}:=\left\langle\phi_{1 j}, \mathcal{L}_{1}\left(\phi_{1 k}\right) M \phi_{1 \ell}\right\rangle, \\
& \left(\Gamma_{2}^{\ell}\right)_{j k}:=\left\langle\phi_{1 j}, \mathcal{L}_{2}\left(\phi_{2 k}\right) C \phi_{2 \ell}\right\rangle,
\end{aligned}
$$

The inner product symbol $\langle v, w\rangle$ here represents an integration along the entire length of the beam of $v^{\top} w$. We further note that to prescribe a preset movement $u(t)$ is to effectively apply a force at base capable of causing the movement, thus

$$
\begin{aligned}
u & =\sum_{i=1}^{n+6} \phi_{1 i}(0) q_{1 i}=G q_{1} \\
\dot{u} & =G \dot{q}_{1}
\end{aligned}
$$

The external forces $\eta_{1}$ can be separated into those resulting from the user-defined force, $f_{1}(s, t)$, and a reaction force at the moving base, $f_{r}(t)$, and formulated as:

$$
\eta_{1}=\eta_{e}+\eta_{r}
$$

with the forcing terms being $\eta_{r, j}=\phi_{1 j}(0)^{\top} f_{r}=F f_{r}$ and $\eta_{e j}=\left\langle\phi_{1 j}, f_{1}\right\rangle$. Now,

$$
\dot{u}=G A_{1}^{-1}\left(B_{1} q_{2}-\left(q_{1 \ell} \Gamma_{1}^{\ell} q_{1}+q_{2 \ell} \Gamma_{2}^{\ell} q_{2}\right)+\eta_{e}\right)+G A_{1}^{-1} F f_{r} .
$$


Solving for $\eta_{r}$,

$$
\eta_{r}=F\left(G A_{1}^{-1} F\right)^{-1} \dot{u}-F\left(G A_{1}^{-1} F\right)^{-1} G A_{1}^{-1}\left(B_{1} q_{2}-\left(q_{1 \ell} \Gamma_{1}^{\ell} q_{1}+q_{2 \ell} \Gamma_{2}^{\ell} q_{2}\right)+\eta_{e}\right) .
$$

The solution of $\eta_{r}$ can be incorporated back into the modal equations:

$$
\dot{q}_{1}=A_{1}^{-1}\left(I-F\left(G A_{1}^{-1} F\right)^{-1} G A_{1}^{-1}\right)\left(B_{1} q_{2}-\left(q_{1 \ell} \Gamma_{1}^{\ell} q_{1}+q_{2 \ell} \Gamma_{2}^{\ell} q_{2}\right)+\eta_{e}\right)+A_{1}^{-1} F\left(G A_{1}^{-1} F\right)^{-1} \dot{u} .
$$

or, collecting terms,

$$
\dot{q}_{1}=A^{*}\left(B_{1} q_{2}-\left(q_{1 \ell} \Gamma_{1}^{\ell} q_{1}+q_{2 \ell} \Gamma_{2}^{\ell} q_{2}\right)+\eta_{e}\right)+A_{1}^{-1} B_{u} \dot{u}
$$

where

$$
A^{*}=A_{1}^{-1}\left(I-F\left(G A_{1}^{-1} F\right)^{-1} G A_{1}^{-1}\right)
$$

and

$$
B_{u}=F\left(G A_{1}^{-1} F\right)^{-1} .
$$

Thus the prescription of base translations transforms the dynamics with an additional $A^{*}$ matrix. This is an augmentation (with initial curvature and base movements) upon the modal equations of Ref. [17, equation (20)]. This choice of the intrinsic formulation as the structural model in this work partially arises from the fact that it is an exact description of the structural dynamics which only contains quadratic nonlinearities. The nonlinear couplings are relatively small even when the structure undergoes significant deformations as will be shown in Fig. 2. These characteristics make the formulation very suitable for the synthesis of a control system.

\section{Aeroelastic Formulation}

The aerodynamic component of the model relies on 2-D thin-strip aerofoil model, where the Wagner's function is used to compute the aerodynamic forces from the aerofoil's local velocities, ${ }^{20}$ and provides a linear aerodynamic description with sufficient accuracy for this study. This model computes the local lift force on a wing section as a sum of the contributions from quasi-steady (instantaneous) angle of attack $\alpha_{q s}$ and circulation-induced angle of attack $\alpha_{i}$ :

$$
\mathbf{f}_{\mathbf{a}}(s)=\rho_{\infty} b v_{y}^{2}\left\{\begin{array}{c}
0 \\
-c_{d 0}-\frac{v_{z}}{v_{y}} c_{l} \\
c_{l}
\end{array}\right\}, \quad \mathbf{m}_{\mathbf{a}}(s)=\rho_{\infty} b^{2} v_{y}^{2}\left\{\begin{array}{c}
a c_{l}-\frac{\pi}{2} \frac{b \omega_{x}}{v_{y}} \\
0 \\
0
\end{array}\right\} .
$$

with

$$
c_{l}=\pi\left(\alpha_{q s}+\alpha_{i}\right),
$$

where $v$ and $\omega$ are local components of velocities and angular velocities.

The quasi-steady component of lift is a function of the inertial velocity vector $\mathbf{x}_{1}$ :

$$
\alpha_{q s}=\frac{b}{v_{y}} \kappa^{T} \mathbf{x}_{1}
$$

where

$$
\boldsymbol{\kappa}^{T}=\left\{\begin{array}{llllll}
0 & 0 & -\frac{1}{b} & (1-a) & 0 & 0
\end{array}\right\} .
$$

The induced component of angle of attack is obtained from a rational-function state-space approximation of the lift deficiency function, $C(k)$, in Theodorsen's airfoil theory. This results in

$$
\alpha_{i}=2 \sum_{n=1}^{N_{a}} A_{n}^{a e} b_{n}^{a e} \lambda_{n}
$$

where the aerodynamic states $\lambda_{n}$ satisfy

$$
\dot{\lambda}_{n}+\frac{b_{n}^{a e} V_{\infty}}{b} \lambda_{n}=\kappa^{T} \mathbf{x}_{1} .
$$


For $N_{a}=2$ aerodynamic states, a good approximation to the values of the non-dimensional constants are $b_{1}^{a e}=0.0455, b_{2}^{a e}=0.3, A_{1}^{a e}=0.165, A_{2}^{a e}=0.335$. The exact form of the aerodynamic forces in the modal description, derived using Wagner's function, is described by Palacios and Epureanu. ${ }^{18}$ These forces can be approximated by a quadratic coupling between the velocity modes augmenting the modal ODE. The aeroelastic modal dynamics can then be written as:

$$
\begin{aligned}
A_{1} \dot{q}_{1} & =B_{1} q_{2}-\left(q_{1 \ell}\left(\Gamma_{1}^{\ell}-\rho_{\infty} \Xi_{1}^{\ell}\right) q_{1}+q_{2 \ell} \Gamma_{2}^{\ell} q_{2}\right)+2 A_{n}^{a e} b_{n}^{a e} \rho_{\infty} \lambda_{n, \ell} \Xi_{2}^{\ell} q_{1}+\eta_{1}, \\
A_{2} \dot{q}_{2} & =B_{2} q_{1}+q_{2 \ell}\left(\Gamma_{2}^{\ell}\right)^{\top} q_{1}, \\
\dot{\lambda}_{n} & =q_{1}-\frac{b_{n}^{a e} V_{\infty}}{b} \lambda_{n} .
\end{aligned}
$$

where $\lambda_{n}$ being a vector of the aerodynamic states and $\Xi$ is given by ${ }^{18}$

$$
\begin{aligned}
& \left(\Xi_{1}^{\ell}\right)_{j k}:=\left\langle\phi_{1 j}, \mathcal{A}_{1}\left(\phi_{1 k}\right) \pi b \phi_{1 \ell}\right\rangle, \\
& \left(\Xi_{2}^{\ell}\right)_{j k}:=\left\langle\phi_{2 j}, \mathcal{A}_{2}\left(\mathbf{x}_{1}(s, 0)\right) \pi b \phi_{1 k} \boldsymbol{\kappa}^{T} \phi_{1 \ell}\right\rangle,
\end{aligned}
$$

where

$$
\mathcal{A}_{1}\left(\mathbf{x}_{1}\right)=\left[\begin{array}{cccccc}
0 & 0 & 0 & 0 & 0 & 0 \\
0 & -\frac{c_{d 0}}{\pi} v_{y} & v_{z} & -b(1-a) v_{z} & 0 & 0 \\
0 & -v_{z} & 0 & b(1-a) v_{y} & 0 & 0 \\
0 & -a b v_{z} & 0 & \frac{2 a-2 a^{2}-1}{2} b^{2} v_{y} & 0 & 0 \\
0 & 0 & 0 & 0 & 0 & 0 \\
0 & 0 & 0 & 0 & 0 & 0
\end{array}\right], \quad \mathcal{A}_{2}\left(\mathbf{x}_{1}\right)=\left[\begin{array}{cccccc}
0 & 0 & 0 & 0 & 0 & 0 \\
0 & 0 & -v_{y} & 0 & 0 & 0 \\
0 & v_{y} & 0 & 0 & 0 & 0 \\
0 & a b v_{y} & 0 & 0 & 0 & 0 \\
0 & 0 & 0 & 0 & 0 & 0 \\
0 & 0 & 0 & 0 & 0 & 0
\end{array}\right]
$$

Note that $\Xi_{2}$ is computed under a fixed reference airspeed, $V_{\infty}$, and thus will change when the reference flight conditions are changed. Any control surface input will enter the model via aerodynamic forces in the $\eta_{1}$ term.

\section{Control Problem Definition}

Sections II and III have described an aeroelastic model as state-space system with quadratic nonlinearity. To synthesise a controller requires the definition of inputs and outputs to the system. In the spirit of the eventual goal of this study, the control system will focus on vibration suppression and gust alleviation. For vibration suppression studies, the disturbances are defined as basal accelerations, and the control inputs will be forces applied on the beam tip. In the aeroelastic problem (Eq. (25)), the disturbance will be assumed to be external gust loads (modelled as a prescribed distributed force), and the control input would be flap deflections at particular spanwise locations.

The $\mathcal{H}_{\infty}$ controller is a linear optimal controller with linear observers and controllers that act to minimise the $\infty$-norm of the transfer function, in effect minimising the all-time maximum of the output caused by a disturbance. ${ }^{22}$ This is important in gust alleviation as it is often a critical requirement to limit the maximum structural load experienced by the wing. However, note that it was shown ${ }^{23}$ that there is not a great difference between the use of $\mathcal{H}_{\infty}$ in continuous disturbance rejection compared to an LQG controller which is specifically optimised for this task.

To synthesise the $\mathcal{H}_{\infty}$ control system, define the system state as

$$
q=\left(\begin{array}{c}
q_{1} \\
q_{2} \\
\lambda_{n}
\end{array}\right),
$$

where $q_{1}$ and $q_{2}$ are the state vectors of velocity and force respectively, and $u$ represents the 6 components of basal velocity. The full open-loop system is written as:

$$
\begin{aligned}
\dot{q} & =M_{A} q+M_{E} d+M_{B} w+\mathcal{F}^{2}(q, d, w), \\
y_{c t r l} & =M_{C_{1}} q+M_{D_{11}} d+M_{D_{12}} w, \\
y_{\text {meas }} & =M_{C_{2}} q+M_{D_{21}} d+M_{D_{22}} w,
\end{aligned}
$$


where $M_{C_{1}}=M_{C_{2}}=M_{C}$ and the $M_{D}$ matrices are zero matrices of the appropriate dimensions, as the disturbance and input do not affect the output directly. The $\mathcal{F}^{2}$ term contains the quadratic couplings between the states and is the only nonlinear term in the equation. In the structural-only case with basal translations as disturbances, $d$ will be a vector containing basal accelerations $\dot{u}$, and $w$ is the control force input. The system matrix $M_{A}$ is defined as the following:

$$
M_{A}=\left(\begin{array}{cc}
A^{*} & 0 \\
0 & 0
\end{array}\right) \cdot\left(\begin{array}{ccc}
0 & A_{1}^{-1} B_{1} & 0 \\
A_{2}^{-1} B_{2} & 0 & 0 \\
0 & 0 & 0
\end{array}\right),
$$

where matrices $A_{i}, B_{i}$ and $A^{*}$ are defined in Eq. (10) and Eq. (17) respectively. Note that $A^{*}$ will be replaced by $I$ when basal accelerations are not prescribed. In the aeroelastic case, the matrix $M_{A}$ is defined by linearising Eq. (25) around a steady flight condition. Define the observed states (for both controlled and measured output) as a sensor measuring velocities and forces at particular locations $s_{j}$ along the beam axis, thus

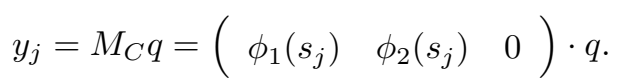

The control input matrix is

$$
M_{B}=\left(\begin{array}{cc}
A^{*} & 0 \\
0 & 0
\end{array}\right) \cdot\left(\begin{array}{c}
\left\langle\phi_{1}, \hat{f}_{j}\right\rangle \\
0 \\
0
\end{array}\right)
$$

where the inner product \langle\rangle is again integration over the entire beam, and $\hat{f}_{j}$ describes normalised input force, which is then scaled using the forcing vector $w$, i.e.:

$$
f_{1}=\sum \hat{f}_{j} \cdot w_{j}
$$

The disturbance enters the system via

$$
M_{E}=\left(\begin{array}{c}
A_{1}^{-1} B_{u} \\
0 \\
0
\end{array}\right)
$$

as prescribed basal accelerations, or,

$$
M_{E}=\left(\begin{array}{c}
\left\langle\phi_{1}, \hat{g}_{j}\right\rangle \\
0 \\
0
\end{array}\right)
$$

as disturbance forces, in a form similar to control forcing, where $\hat{g}_{j}$ describes the normalised disturbance force. In the current form the linear part of the equation (without the $\mathcal{F}^{2}$ term) can be used to synthesise the $\mathcal{H}_{\infty}$ controller, which takes the form of:

$$
\begin{aligned}
\dot{z} & =K_{A} z+K_{B} y_{\text {meas }}, \\
w & =K_{C} z+K_{D} y_{\text {meas }} .
\end{aligned}
$$

where the controller dynamics are given by the $K$ matrices and $z$ is the vector containing the controller states. As the structural eigenmodes are orthogonal and energy-preserving, the eigenvalues of the open-loop system lie entirely on the imaginary axis. Therefore, as a requirement in the synthesis of the $\mathcal{H}_{\infty}$ controller, the eigenvalues are shifted into the negative half-plane by adding $(-\epsilon \cdot I)$ to $M_{A}$ where the value of $\epsilon$ is chosen to be $10^{-3}$ of the smallest eigenvalue of $M_{A}$. Although the addition of aerodynamics tend to damp most poles, this modification is still necessary as long as the open-loop dynamics contain undamped modes, e.g. axial modes. It should also be noted that in order to tune the controller, the system is further augmented with tuning parameters in the form of:

$$
\begin{aligned}
& \bar{M}_{D_{12}}=\left(\begin{array}{c}
M_{D_{12}} \\
k_{w}
\end{array}\right), \\
& \bar{M}_{D_{21}}=\left(\begin{array}{ll}
M_{D_{21}} & k_{d}
\end{array}\right),
\end{aligned}
$$


as the variables $\bar{y}_{c t r l}$ and $\bar{d}$ are augmented to become:

$$
\begin{aligned}
\bar{y}_{c t r l} & =\left(\begin{array}{c}
y_{c t r l} \\
y_{w}
\end{array}\right), \\
\bar{d} & =\left(\begin{array}{c}
d \\
d_{\text {meas }}
\end{array}\right),
\end{aligned}
$$

as part of the standard treatment of controller synthesis. Note that the original $M_{D_{12}}$ and $M_{D_{21}}$ are zero matrices. Here $k_{w}$ places a weight on the input $w$ so that the controller does not respond with an unrealistically large input, whereas $k_{d}$ places a weight on the sensor measurement to represent the measurement uncertainties from real-life sensors. The addition of $y_{w}$ and $d_{\text {meas }}$ does not affect the dynamics of the original system in any way.

The $\mathcal{H}_{\infty}$ control synthesis, used with the control problem posed in the way described, tends to create very high frequency poles for the controller which are unrealistic and also have a negative impact to the robustness, or sensitivity of the controller to deviations from the model. Thus loop-shaping ${ }^{24}$ is also conducted in order to tune the controller in the frequency domain and obtain a more desirable response. The technique also ensures that high-frequency modes including unmodelled ones are not overly excited.

\section{Numerical Results}

In this section, numerical results will be presented for the verification of the structure, control and aerodynamic system respectively. The systems are then coupled in order to simulate the response of a cantilever wing moving in freestream. In the solution process, the modal ODE presented in previous sections is marched in time using a Runge-Kutta scheme with automatic timestepping at a relative error tolerance of $0.1 \%$. This numerical marching scheme is to be used in all subsequent simulations. In the modal description, the structural modes used is chosen according to the lowest eigenvalues and limited to prescribed mode types (axial, torsion, in-plane bending etc) whenever these are clearly identifiable.

\section{A. Structural Model Verification}

The initial numerical model (vibrations in vacuo, i.e. without aerodynamic forces) is based on the modal formulation presented in Eq. (9). The beam used in all simulations presented in this subsection is a $50 \times 1 \times 0.5 \mathrm{~m}$ cantilever beam with mass density $8000 \mathrm{kgm}^{-3}$, Young's modulus 200 GPa and Poisson's ratio of 0.3 , modelled under Euler-Bernoulli assumptions where $C=\left[\frac{1}{E A}, 0,0, \frac{1}{G J}, \frac{1}{E I_{2}}, \frac{1}{E I_{3}}\right]$ and $M=$ $\left[\rho A, \rho A, \rho A, \rho I_{1}, \rho I_{2}, \rho I_{3}\right]$.

The test case for validation is chosen to be sinusoidal basal translations on the $r_{1}$ and $r_{3}$ (axial and out-of-plane) axes with $v=v_{\max } \sin (\omega t)$, amplitude $v_{\max }=5 \mathrm{~ms}^{-1}$ at $\omega=1 \mathrm{rad} \mathrm{s}^{-1}$ and was compared against results from a FEM software package (ABAQUS, 200 1-D beam elements simulated with a timestep of $0.02 \mathrm{~s})$. This excitation produces a tip displacement of the order of the beam length $(50 \mathrm{~m})$ and is therefore highly nonlinear. Agreement is good with 15 bending and 10 longitudinal modes used in the simulation, as shown in Fig. 1. Convergence with a lower number of modes is demonstrated in Table 1 . The results show that with 6 modes in both bending and longitudinal directions, the simulated open-loop response is already very close to the one with 15 bending and 10 axial modes, and in turn in good agreement with FEM results. It should be noted that the inclusion of axial modes is necessary as they provide the coupling between bending modes which are otherwise isolated from each other. Although torsional modes are not needed in this particular example as this is a 2-D problem.

It should be noted that in the intrinsic modal description of beams, the nonlinear coupling between modes is much weaker than in a displacement-based description, and for this reason the linear controller performs well on the structural model under relatively large excitations (e.g. tip displacement of $30 \%$ beam length) as the nonlinear structural couplings remained small. To demonstrate the linearity of the formulation, the nonlinear coupling was turned off in the validation model, and the results showed that the transverse tip velocity is still simulated with some accuracy as seen in Fig. 2. However the axial velocity component (not shown) presents larger errors since there is no coupling involved. It should be noted that the controlled response is less severe than the open-loop and thus demonstrates even less nonlinear behaviour. 


\begin{tabular}{|c|c|c|}
\hline Modes & Error $v_{1}$ & Error $v_{3}$ \\
\hline 5 Bending, 5 Axial & $5.95 \%$ & $2.29 \%$ \\
6 Bending, 6 Axial & $5.90 \%$ & $2.27 \%$ \\
10 Bending, 5 Axial & $5.92 \%$ & $2.29 \%$ \\
10 Bending, 10 Axial & $0.081 \%$ & $0.022 \%$ \\
\hline
\end{tabular}

Table 1: Percentage RMS errors in tip velocity history for different mode selections against the case of 15 bending and 10 longitudinal modes.

\section{B. Aeroelastic Model Verification}

The Goland wing mode ${ }^{25}$ is used to validate the coupling between structural and aerodynamic forces in the model. The Goland wing is a low aspect ratio wing in a cantilever configuration and is a well-studied benchmark numerical test case for aeroelastic simulations in which the structural model is based on beam elements. Properties of the beam can be found in Table 2.

\begin{tabular}{|c|c||c|c|}
\hline Chord, $2 b$ & $1.8288 \mathrm{~m}$ & Mass per unit length, $\rho A$ & $35.71 \mathrm{kgm}{ }^{-1}$ \\
Semi-span, $L$ & $6.096 \mathrm{~m}$ & Moment of inertia around e.a., $\rho I_{1}$ & $8.64 \mathrm{~kg} \cdot \mathrm{m}$ \\
Elastic axis (from l.e.) & $0.66 b$ & Torsional stiffness, $G J$ & $0.99 \times 10^{6} \mathrm{~N} \cdot \mathrm{m}^{2}$ \\
C.G. (from l.e.) & $0.86 b$ & Bending stiffness, $E I_{2}$ & $9.77 \times 10^{6} \mathrm{~N} \cdot \mathrm{m}^{2}$ \\
\hline
\end{tabular}

Table 2: Relevant properties of the Goland wing ${ }^{18}$

The airspeed at which flutter occurs on the Goland wing is computed by a linearisation of the dynamics over a range of increasing airspeeds. An unstable eigenvalue in the linearised dynamics indicates that flutter has occurred. The current study uses 11 bending modes and 1 axial mode which produced results that match well with previous studies using 2D aerodynamic approximations (see Table 3), whereas 3D aerodynamic methods such as UVLM more accurately reflect tip effect which have a noticeable impact on the computed flutter speed. ${ }^{18}$

Time-domain simulation shows that structural nonlinearities do not give rise to any limit-cycle oscillations on the Goland wing at post-flutter speeds. Patil et $\mathrm{al}^{26}$ also confirmed that structural nonlinearities in themselves are unable to create limit-cycle oscillations post-flutter. Instead they observed that in general stall and other nonlinear aerodynamic effects will set in well before significant structural nonlinearity is observed.

\begin{tabular}{|c|c|c|c|}
\hline Author & Model & $V_{f}, \mathrm{~ms}^{-1}$ & $\omega_{f}, \mathrm{rad} s^{-1}$ \\
\hline Current & Intrinsic / 2D aero (Modal) & 139 & 70.0 \\
Palacios et al & Intrinsic / 2D aero (Modal) & 141 & 69.8 \\
Sotoudeh et al $^{7}$ & Intrinsic / 2D aero (FD) & 137 & 70.1 \\
Wang et al & Intrinsic / UVLM & 164 & - \\
Murua et al & Displacement / UVLM & 165 & 69 \\
\hline
\end{tabular}

Table 3: Flutter velocity and frequency for the Goland wing at $\rho_{\infty}=1.02 \mathrm{~kg} \mathrm{~m}^{-3}$

\section{Control Action on Structural Model}

Different disturbance scenarios are used to test the efficiency of the controller using the setup described in Section A, which is synthesised to minimise the local transverse velocity of the tip (relative to the global coordinates) in the $r_{3}$ axis $\left(x_{1,3}(L)\right)$ by applying a transverse tip force in the local $r_{3}$ direction. The control synthesis used 6 bending and 6 longitudinal modes in the linearised system and was then applied to a system 
with full nonlinear coupling in the dynamics. The first case was a sinusoidal transverse base velocity of amplitude $5 \mathrm{~ms}^{-1}$ at $1 \mathrm{~Hz}$. Here the closed-loop system demonstrates that the controller was able to reduce the amplitude of the resulting response, with a lower weight on the input allowing for greater amplitude reduction at the expense of a larger controller input (Fig. 3 and 4). A similar result was obtained when applying a white noise disturbance with a frequency cutoff at the highest bending mode eigenvalue of $\omega=86.1$ $\operatorname{rad~s}^{-1}$ (Fig. 5).

For a "1 - sin" excitation (Fig. 6), a lower weight on control input allows the controller to provide a larger input to suppress the excitation, resulting in the disturbance being dampened faster as well as producing a smaller maximum response.

\section{Aeroelastic System with Robust Controller}

The Goland wing has a relatively low aspect ratio and the dynamics observed on the wing are very low in their degree of non-linearity. In order to better study the problems facing a high-aspect ratio wing, the problem is modified so that large deformations can be observed. The modified wing is defined as 5 times as long as the original while possessing only one third of the original stiffness. The flutter speed of this modified wing is found to be $V_{f}=59 \mathrm{~ms}^{-1}$. Fig. 7 shows that a 10-mode system (of which 9 are bending/torsional modes and 1 is axial) is able to reach convergence on the open-loop response. Each structural mode is associated with 2 aerodynamic modes, making the total number of aerodynamic modes 20 . This number of modes will be used in all subsequent simulations.

A linear $\mathcal{H}_{\infty}$ controller is applied on the aeroelastic model in order to observe the effect of gust load alleviation and flutter suppression via active control on the modified wing. The problem is posed so that the controller aims to control the base bending moment, by measuring the base moment and manipulating a control surface of $20 \%$ chord length and mounted on $60 \%$ to $90 \%$ of wing span. The model simulates the base of the cantilever wing moving at $V_{\infty}$ and the controller dynamics is synthesised using a model linearized about the the the current $V_{\infty}$.

It should be noted that the disturbance is not an actual gust but a time-varying distributed force applied on the local transverse out-of-plane direction, where $F(s, t)=(1-\cos (\omega t)) / 2 \cdot F_{\text {Max }}$. This disturbance is chosen due to its simplicity of incorporation into the model as its only purpose is to provide a large enough transient excitation to the structure. A more realistic gust profile only serves the same purpose however requires much more complex treatment but only serves the same purpose.

Simulations using a 1 - cos force profile show that the controller achieves gust load alleviation under a range of gust lengths (Fig. 8), with a $8.3 \%$ reduction in maximum root bending moment when $\omega=5 \mathrm{rad}$ $\mathrm{s}^{-1}$. Up to a tip deflection of $13.3 \mathrm{~m}$ (Fig. 11), or more than $40 \%$ beam length, no significant deviation from linear behaviour is observed, as shown in Fig. 9 comparing the response to that of a much smaller disturbance, further demonstrating the weak nonlinear coupling of the formulation. A limit to the current model is that control saturation is not implemented, therefore the disturbance is chosen so that the control surface deflection, shown in Fig. 10, is limited to 20 degrees in order to prevent stall from occurring.

When $V_{\infty}$ is chosen to be beyond flutter speed, the controller additionally provides active flutter stabilisation of the wing. Fig. 12 and Fig. 13 shows that the stabilisation effect at $V_{\infty}=65 \mathrm{~ms}^{-1}$ is still relatively robust despite demonstrating a very different response to that of $V_{\infty}=55 \mathrm{~ms}^{-1}$. The system remains stabilised under a large tip deflection (Fig. 14), although again care needs to be taken to limit the flap deflection so that the current model remains a realistic representation of the dynamics.

It should be noted that currently different controllers are designed around their respective $V_{\infty}$, thus how much change in flight speed can be accommodated by a particular controller remains to be seen.

\section{Conclusions}

The computational results demonstrate that a linear robust control design functions very well on the setup investigated in this work, up to a deformation large enough to observe significant geometric nonlinearities. The low degree of coupling between modes in this formulation also serves to explain the good performance of linear controllers synthesised on a displacement-based formulation, despite the large degree of nonlinearity involved in which.

As HALE aircraft normally operate at a safe margin from stall conditions, control saturation is likely to be the major contributor to more complex dynamic behaviour of the wing. The current model also lacks 
full rigid body degrees of freedom, the incorporation of which is also likely to have a major impact on the dynamics and possibly give rise to further nonlinear effects.

Future work will also investigate a more efficient incorporation of displacement into the intrinsic model which allows modelling of gravity, and a multi-beam configuration which allows a full aircraft configuration to be studied. The robustness of the controller when subject to different airspeeds $V_{\infty}$ will also be studied.

\section{Acknowledgements}

This work is funded by the UK Engineering and Physical Sciences Research Council Grant EP/I014683/1 "Nonlinear Flexibility Effects on Flight Dynamics and Control of Next-Generation Aircraft". This support is gratefully acknowledged.

\section{References}

${ }^{1}$ Lucia, D., "The SensorCraft Configurations- A Non-Linear AeroServoElastic Challenge for Aviation," 46 th AIAA/ASME/ASCE/AHS/ASC Structures, Structural Dynamics, and Materials Conference, 2005, pp. 1-7.

${ }^{2}$ Cesnik, C., Senatore, P., Su, W., Atkins, E., and Shearer, C., "X-HALE: A Very Flexible Unmanned Aerial Vehicle for Nonlinear Aeroelastic Tests," AIAA Journal, Vol. 50, No. 12, December 2012, pp. 2820-2833.

${ }^{3}$ Burnett, E., Atkinson, C., Beranek, J., Sibbitt, B., Holm-Hansen, B., and Nicolai, L., "NDOF Simulation Model for Flight Control Development with Flight Test Correlation," AIAA Modeling and Simulation Technologies Conference, Toronto, Canada, August 2010, AIAA Paper 2010-7780.

${ }^{4}$ Drela, M., "Integrated simulation model for preliminary aerodynamic, structural, and control-law design of aircraft," $40^{\text {th }}$ AIAA/ASME/ASCE/AHS/ASC Structures, Structural Dynamics and Materials Conference, St. Louis, Missouri, USA, 1999, AIAA Paper 1999-1394.

${ }^{5}$ Patil, M., Hodges, D., and Cesnik, C., "Nonlinear aeroelasticity and flight dynamics of high-altitude long-endurance aircraft," Journal of Aircraft, Vol. 38, No. 1, 2001, pp. 88-94.

${ }^{6}$ Brown, E., Integrated strain actuation in aircraft with highly flexible composite wings, Ph.D. thesis, Massachusetts Institute of Technology, 2003.

${ }^{7}$ Sotoudeh, Z., Hodges, D., and Chang, C., "Validation Studies for Aeroelastic Trim and Stability Analysis of Highly Flexible Aircraft," Journal of Aircraft, Vol. 47, No. 4, 2010, pp. 1240-1247.

${ }^{8}$ Wang, Z., Chen, P. C., Liu, D. D., and Mook, D. T., "Nonlinear-Aerodynamics/Nonlinear-Structure Interaction Methodology for a High-Altitude Long-Endurance Wing," Journal of Aircraft, Vol. 47, No. 2, 2010, pp. 556-566.

${ }^{9}$ Murua, J., Palacios, R., and Graham, J., "Applications of the unsteady vortex-lattice method in aircraft aeroelasticity and flight dynamics," Progress in Aerospace Sciences, Vol. 55, Nov. 2012, pp. 46-72.

${ }^{10}$ Cook, R. G., Palacios, R., and Goulart, P., "Robust Gust Alleviation and Stabilization of Very Flexible Aircraft," AIAA Journal, Vol. 51, No. 2, February 2013, pp. 330-340.

${ }^{11}$ Dillsaver, M., Cesnik, C., and Kolmanovsky, I., "Gust Load Alleviation Control for Very Flexible Aircraft," AIAA Atmospheric Flight Mechanics Conference, Minneapolis, Minnesota, August 2011.

${ }^{12}$ Wagg, D. and Neild, S., "A Review of Non-Linear Structural Control Techniques," Proceedings of the Institution of Mechanical Engineers, Part C: Journal of Mechanical Engineering Science, Vol. 225, No. 4, April 2011, pp. 759-770.

${ }^{13}$ Strganac, T., Ko, J., Thompson, D., and Kurdila, A., "Identification and control of limit cycle oscillations in aeroelastic systems," Journal of Guidance Control and Dynamics, Vol. 23, No. 6, 2000, pp. 1127-1133.

${ }^{14}$ Maschke, B., Ortega, R., and Van Der Schaft, A. J., "Energy-based Lyapunov functions for forced Hamiltonian systems with dissipation," IEEE Transactions on Automatic Control, Vol. 45, No. 8, 2000, pp. 1498-1502.

${ }^{15}$ Macchelli, A., "Energy shaping of distributed parameter port-Hamiltonian systems based on finite element approximation," Systems \& Control Letters, Vol. 60, No. 8, 2011, pp. $579-589$.

${ }^{16}$ Hodges, D., "Geometrically exact, intrinsic theory for dynamics of curved and twisted anisotropic beams," AIAA Journal, Vol. 41, No. 6, 2003, pp. 1131-1137.

${ }^{17}$ Palacios, R., "Nonlinear normal modes in an intrinsic theory of anisotropic beams," Journal of Sound and Vibration, Vol. 330, No. 8, April 2011, pp. 1772-1792.

${ }^{18}$ Palacios, R. and Epureanu, B., "An Intrinsic Description of the Nonlinear Aeroelasticity of Very Flexible Wings," 52th AIAA/ASME/ASCE/AHS/ASC Structures, Structural Dynamics, and Materials Conference, Denver, Colorado, USA, April 2011.

${ }^{19}$ Wynn, A., Palacios, R., Goulart, P., and Wang, Y., "Conserved Quantities in Nonlinear Beam Vibrations: An Intrinsic Approach," Submitted to Journal of Sound and Vibration.

${ }^{20}$ Palacios, R., Murua, J., and Cook, R., "Structural and Aerodynamic Models in Nonlinear Flight Dynamics of Very Flexible Aircraft," AIAA Journal, Vol. 48, No. 11, 2010, pp. 2648-2659.

${ }^{21}$ Hodges, D., "Nonlinear Composite Beam Theory," Progress in Astronautics and Aeronautics, Vol. 213, 2006.

${ }^{22}$ Fairman, F., Linear Control Theory, the State Space Approach, Wiley, 1998.

${ }^{23} \mathrm{Ng}$, B., Palacios, R., Graham, M., and Kerrigan, E., "Robust control synthesis for gust load alleviation from large aeroelastic models with relaxation of spatial discretisation," EWEA 2012, Copenhagen, Denmark, 2012.

${ }^{24}$ Kwakernaak, H. and Sivan, R., Linear Optimal Control Systems, Wiley, 1972.

${ }^{25}$ Goland, M., "The Flutter of a Cantilever Wing," Journal of Applied Mechanics, Vol. 12, No. 4, 1945, pp. $197-208$. 
${ }^{26}$ Patil, M. J., Hodges, D. H., and Cesnik, C. E., "Limit-cycle oscillations in high-aspect-ratio wings," Journal of Fluids and Structures, Vol. 15, No. 1, 2001, pp. 107-132.

${ }^{27}$ Wang, Z., Chen, P., Liu, D., and Mook, D., "Nonlinear-aerodynamics/nonlinear-structure interaction methodology for a high-altitude long-endurance wing," Journal of Aircraft, Vol. 47, No. 2, 2010, pp. 556-566.

${ }^{28}$ Murua, J., Palacios, R., and Graham, J., "Assessment of Wake-Tail Interference Effects on the Dynamics of Flexible Aircraft," AIAA Journal, Vol. 50, No. 7, 2012, pp. 1575-1585. 


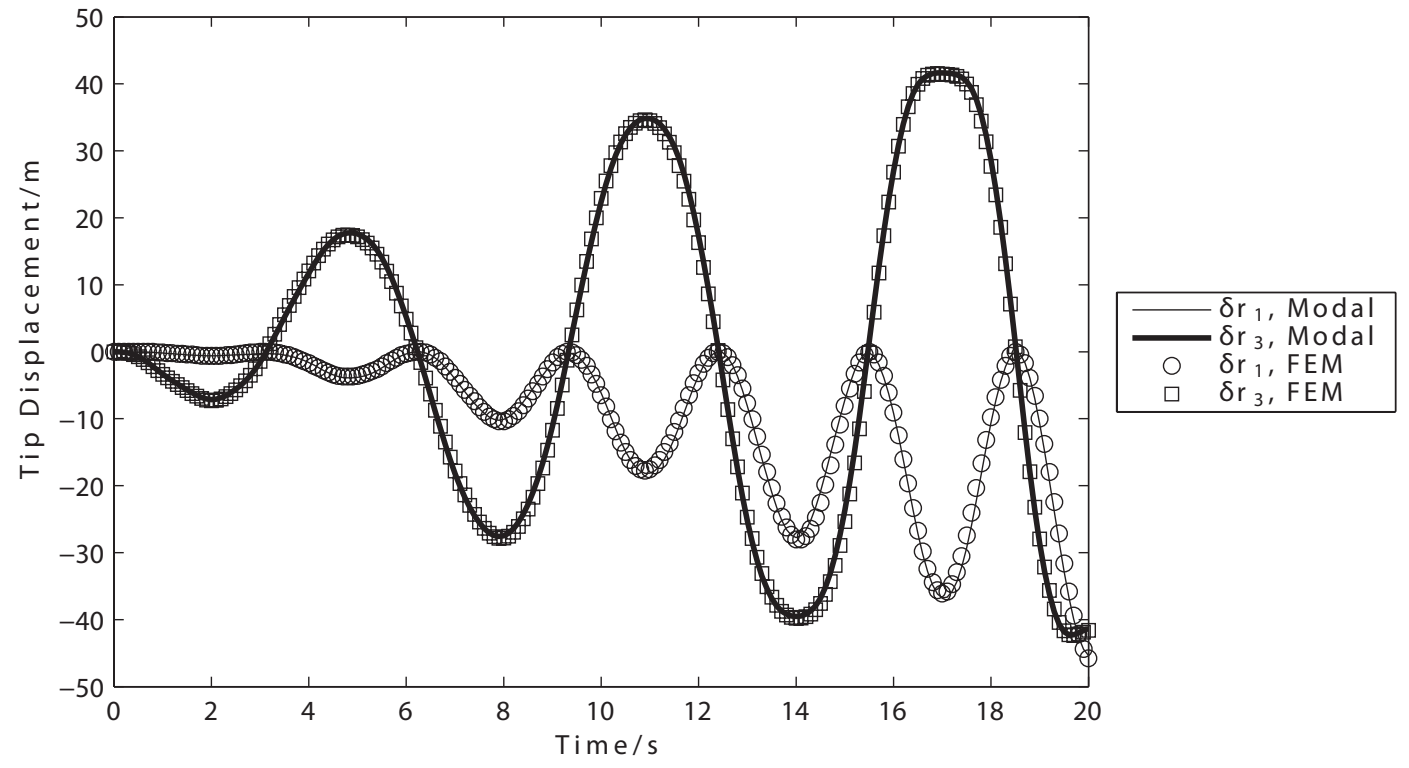

Figure 1: Validation study on the $50 \mathrm{~m}$ beam using a sinusoidal base velocity excitation of $5 \mathrm{~ms}^{-1}$ amplitude in the $r_{1}$ and $r_{3}$ directions using 15 bending and 10 longitudinal modes, plotted against FEM results. 


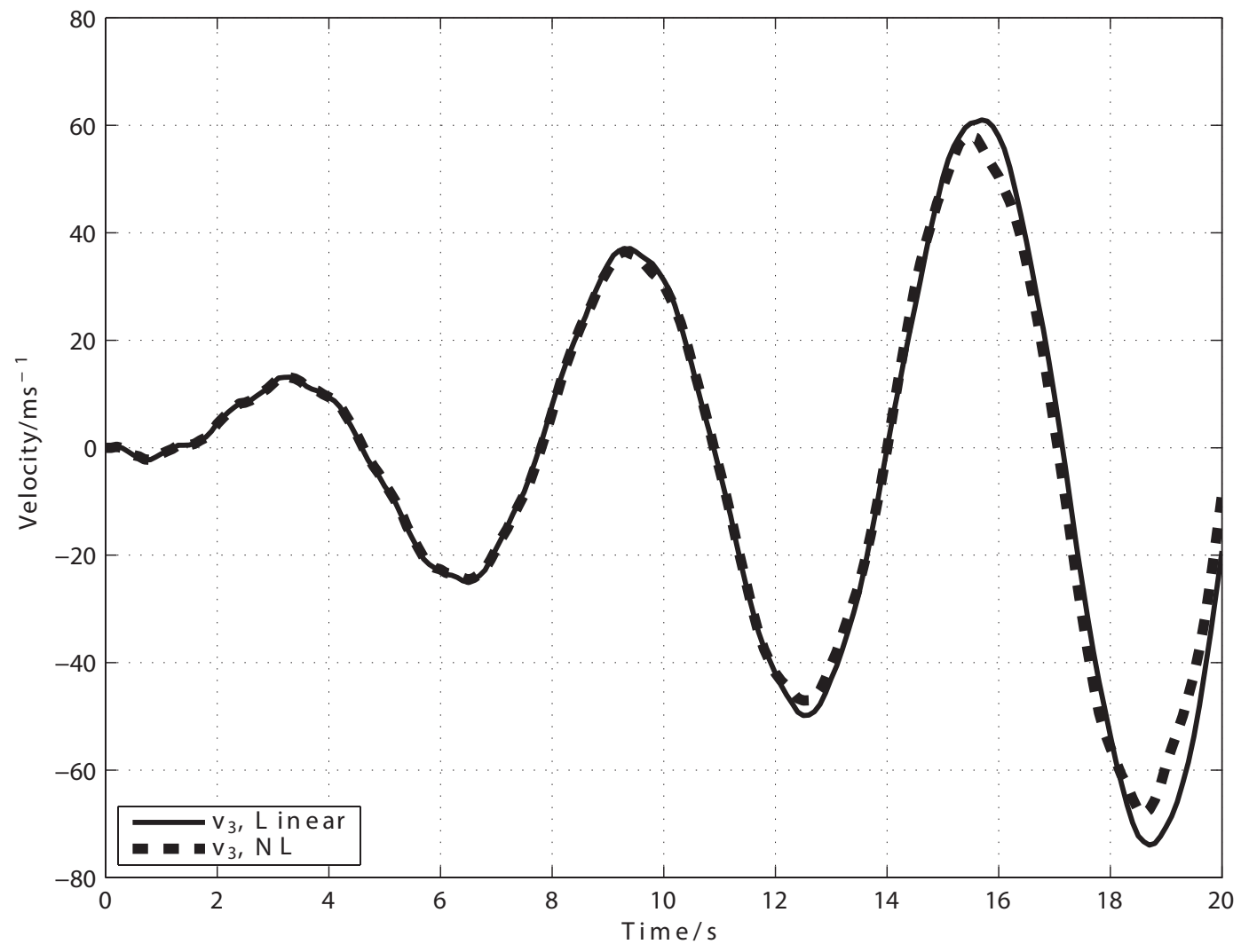

Figure 2: A comparison of the computed transverse tip velocity for the base velocity excitation of $5 \mathrm{~ms}^{-1}$ amplitude in the $r_{1}$ and $r_{3}$ directions on the $50 \mathrm{~m}$ beam using 15 bending and 10 longitudinal modes, with and without nonlinear couplings between modes. It can be seen that even when the deformation is in the order of $L$, the linear approximation still performs well in the transverse direction. 


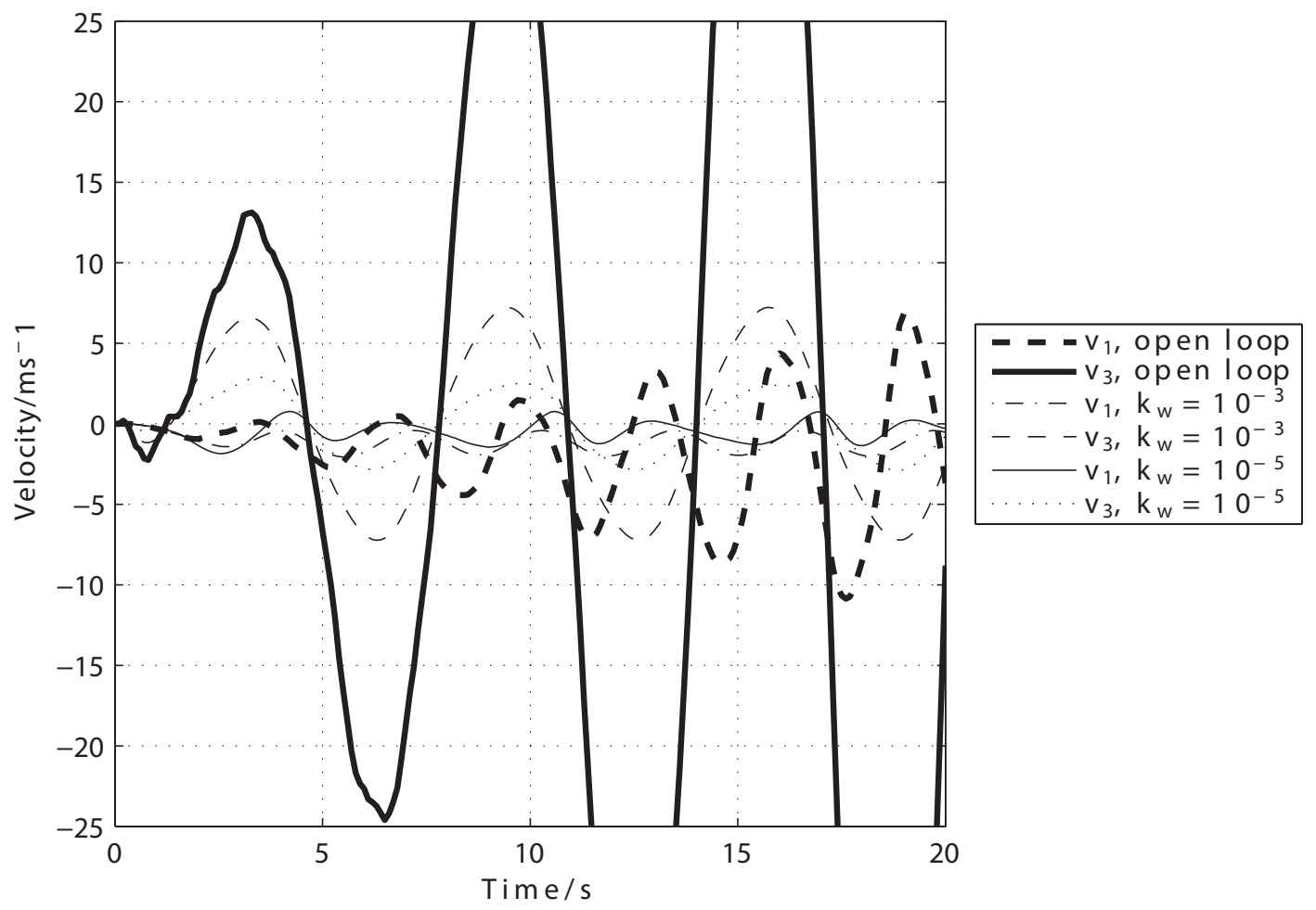

Figure 3: The effect of tip force control input in suppressing the tip velocity when subject to the continuous sinusoidal excitation of $5 \mathrm{~ms}^{-1}$ amplitude at $1 \mathrm{~Hz}$ in $r_{1}$ and $r_{3}$ axis on the $50 \mathrm{~m}$ beam, with different weights used in control synthesis. 


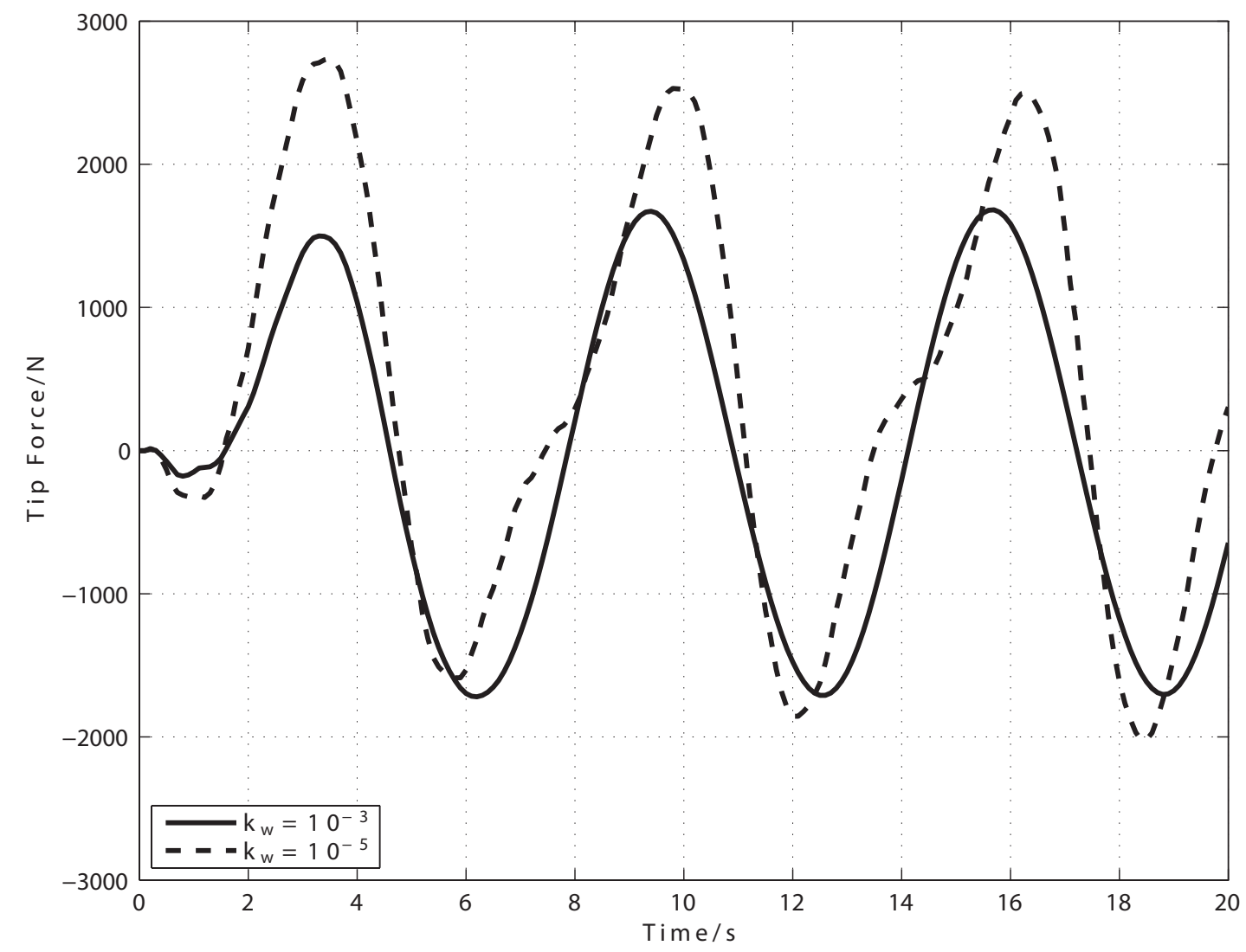

Figure 4: Tip force controller input when subject to the continuous sinusoidal excitation of $5 \mathrm{~ms}^{-1}$ amplitude at $1 \mathrm{~Hz}$ in $r_{1}$ and $r_{3}$ axis on the $50 \mathrm{~m}$ beam, with different weights used in control synthesis. 


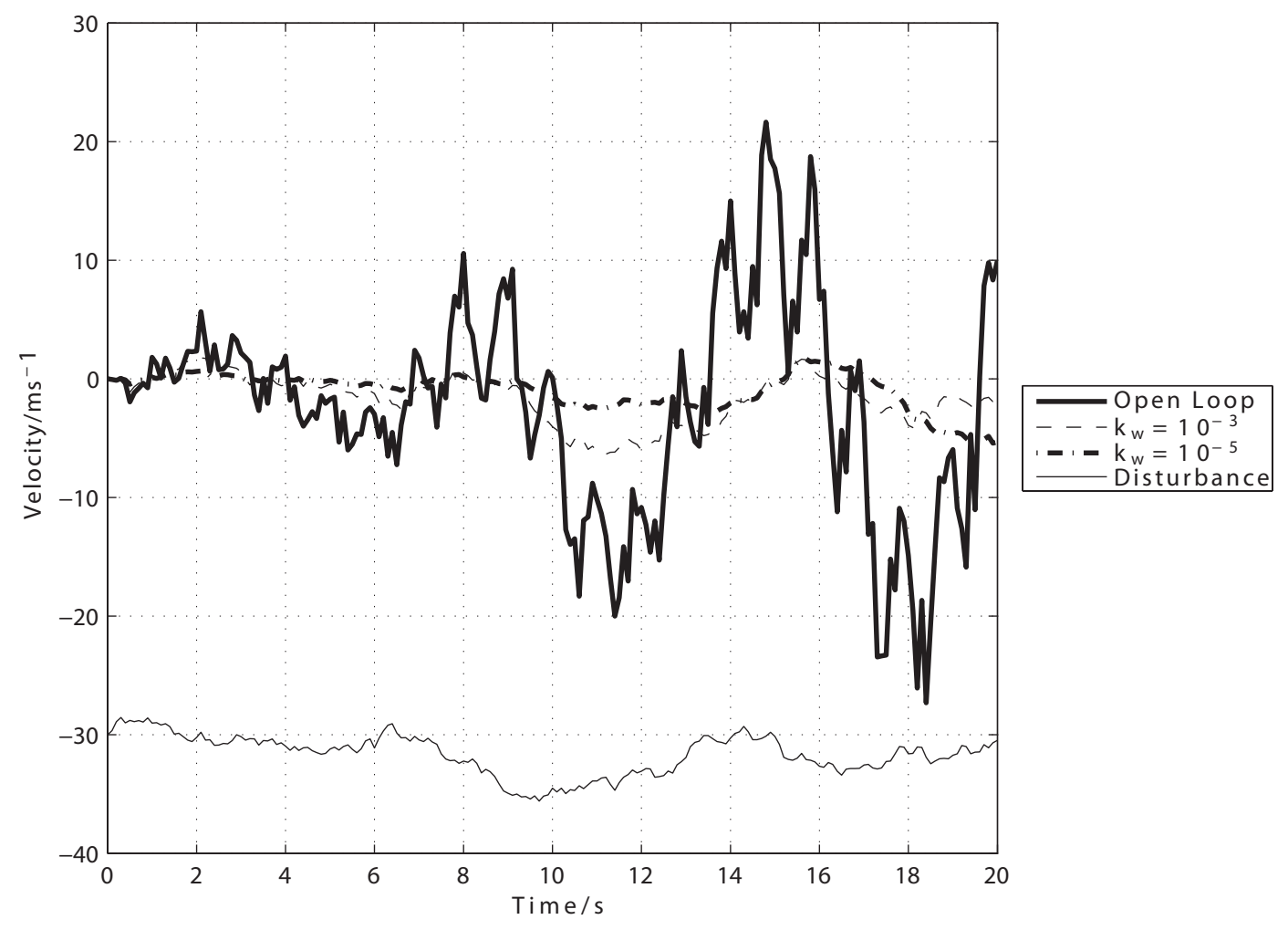

Figure 5: The effect of tip force control input in suppressing the tip velocity when subject to a white noise base excitation of $\dot{\bar{u}}=10 \mathrm{~ms}^{-2}$ on the $50 \mathrm{~m}$ beam, with different weights used in control synthesis.

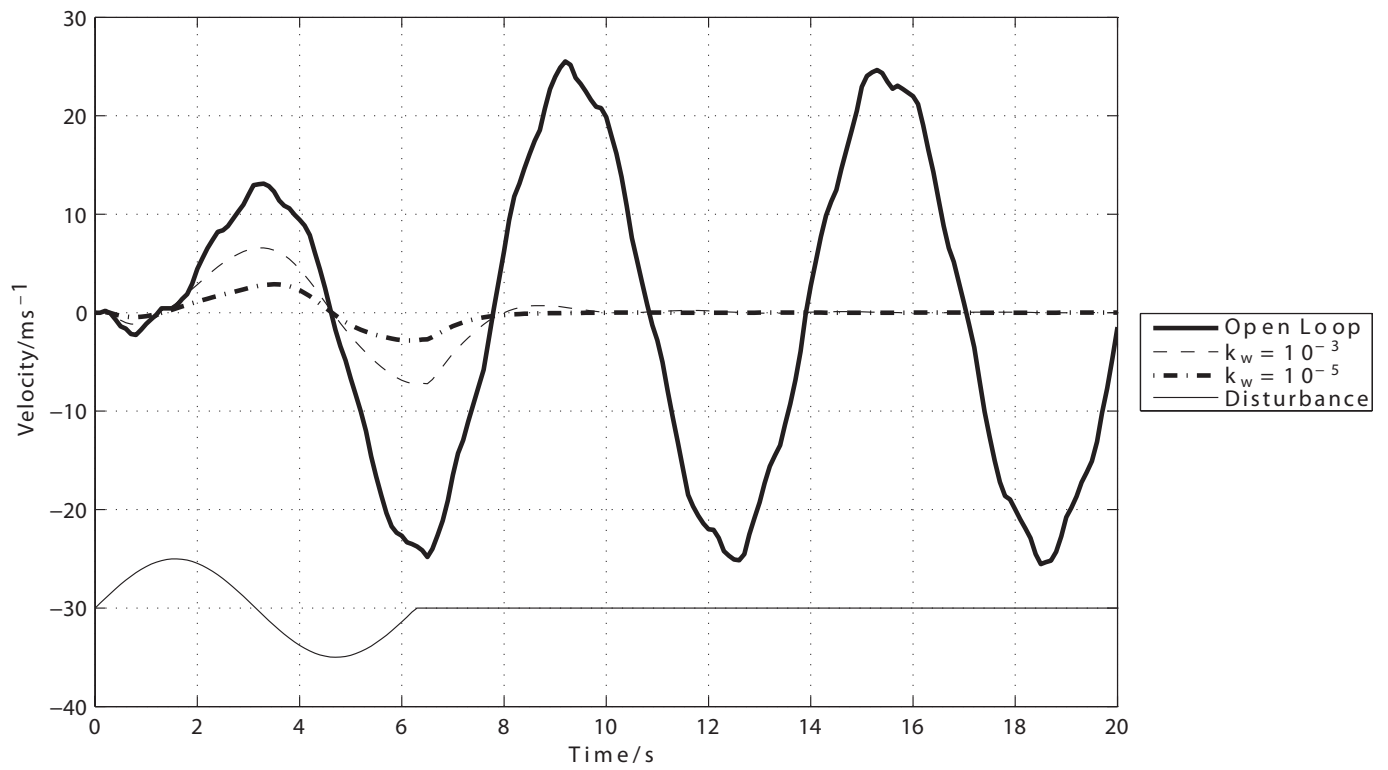

Figure 6: The effect of tip force control input in suppressing the tip velocity when subject to a 1-cycle sinusoidal excitation of $5 \mathrm{~ms}^{-1}$ amplitude on the $50 \mathrm{~m}$ beam, with different weights used in control synthesis. 


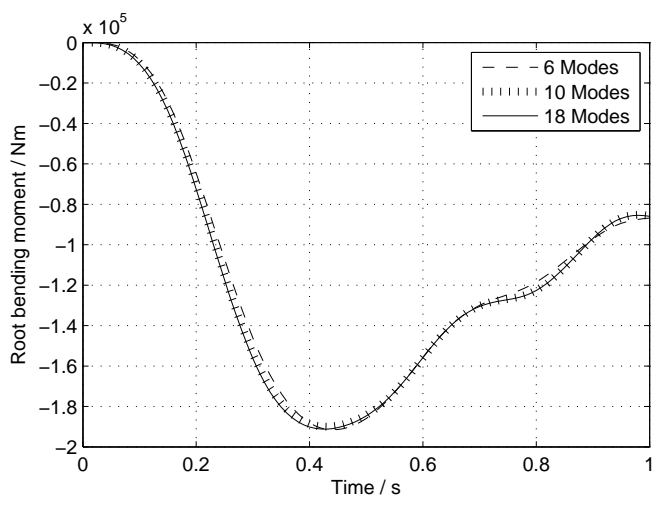

Figure 7: Convergence of root bending moment at $V_{\infty}=55 \mathrm{~ms}^{-1}$ on the modified wing. Shown with a 1-cos distributed force of $F_{M a x}=15000 \mathrm{Nm}^{-1}$ on the local transverse direction with $\omega=5 \mathrm{rad} \mathrm{s}^{-1}$. Computed with 6,10 and 18 lowest frequency modes.

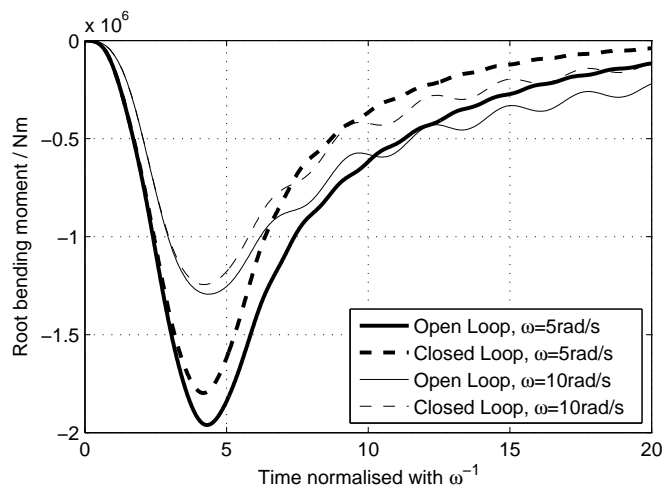

Figure 8: Open- and closed-loop root bending moment on the modified wing when a 1-cos distributed force of $F_{\text {Max }}=10000 \mathrm{Nm}^{-1}$ is applied at $V_{\infty}=55 \mathrm{~ms}^{-1}$.

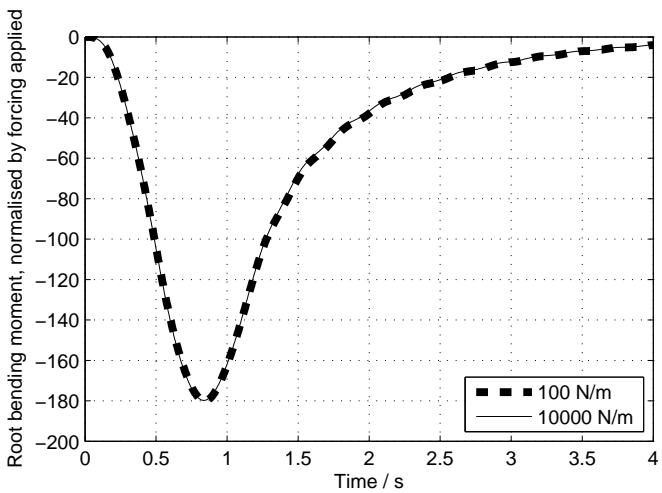

Figure 9: Closed-loop root bending moment variation on the modified wing under different values of $F_{\text {Max }}$, normalised with $F_{\text {Max }}$. Simulated under $V_{\infty}=55 \mathrm{~ms}^{-1}$ and demonstrating of linearity of the problem. Disturbance is a 1-cos distributed force on the local transverse direction with $\omega=5 \mathrm{rad} \mathrm{s}^{-1}$. 


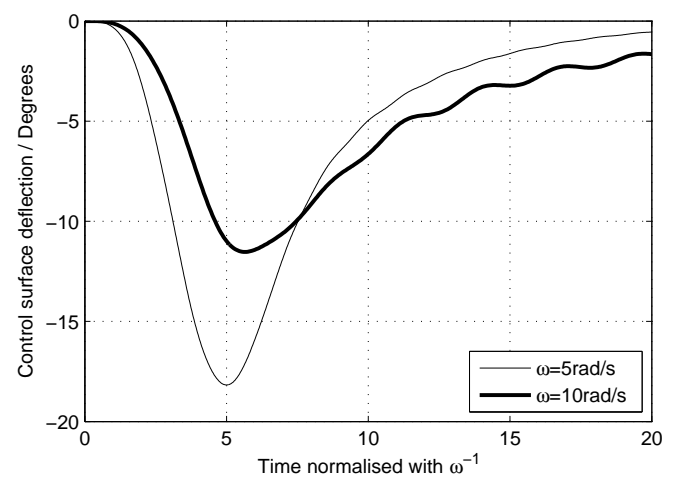

Figure 10: Control surface deflection on the modified wing when a 1-cos distributed force of $F_{\text {Max }}=10000$ $\mathrm{Nm}^{-1}$ is applied at $V_{\infty}=55 \mathrm{~ms}^{-1}$.

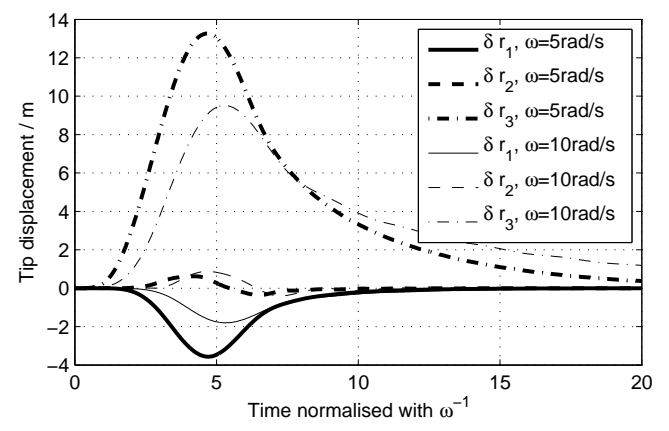

Figure 11: Tip deflection on the modified wing when a 1-cos distributed force of $F_{\text {Max }}=10000 \mathrm{Nm}^{-1}$ is applied at $V_{\infty}=55 \mathrm{~ms}^{-1}$.

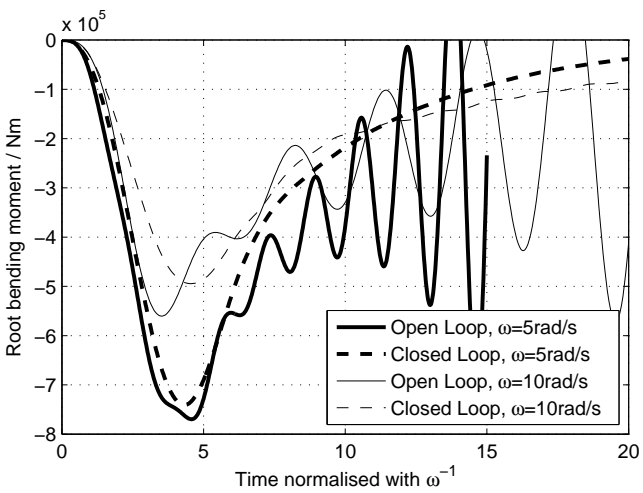

Figure 12: Open- and closed-loop root bending moment on the modified wing when a 1-cos distributed force of $F_{\text {Max }}=5000 \mathrm{Nm}^{-1}$ is applied at $V_{\infty}=65 \mathrm{~ms}^{-1}$. 


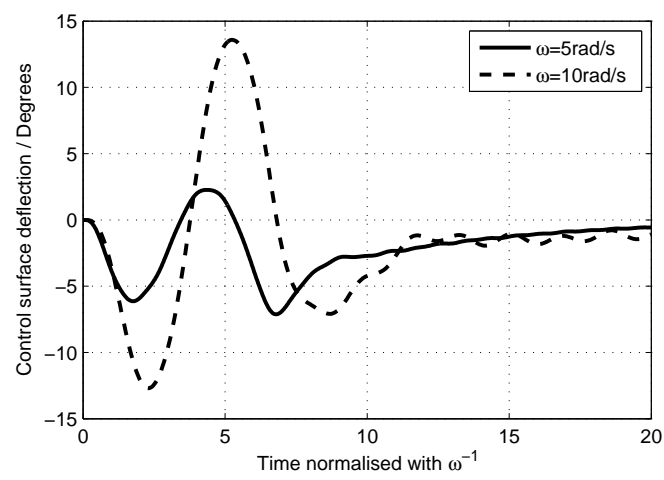

Figure 13: Control surface deflection on the modified wing when a 1-cos distributed force of $F_{M a x}=5000$ $\mathrm{Nm}^{-1}$ is applied at $V_{\infty}=65 \mathrm{~ms}^{-1}$.

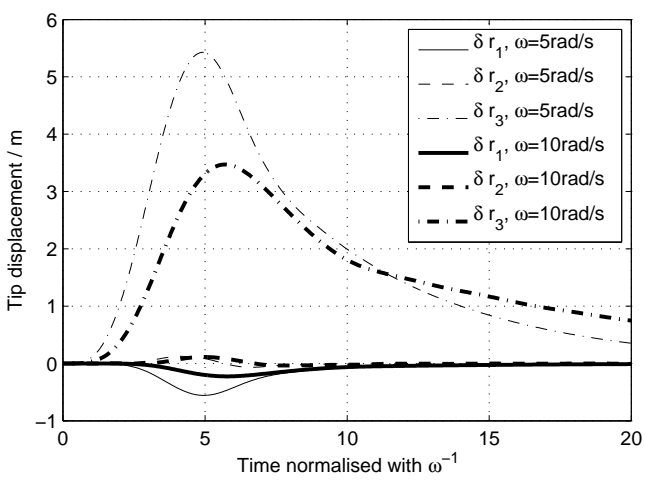

Figure 14: Tip deflection on the modified wing when a 1-cos distributed force of $F_{M a x}=5000 \mathrm{Nm}^{-1}$ is applied at $V_{\infty}=65 \mathrm{~ms}^{-1}$. 